\section{Assessment of the accuracy of ultrasound compared to magnetic resonance imaging in the ability to detect metasta- tic breast cancer to the axilla}

\author{
Sarah Ines Ramirez, Max Scholle, \\ Jennifer Buckmaster, \\ Gopal Chandru Kowdley \\ Department of Surgery, Saint Agnes \\ Hospital, Baltimore, USA
}

\begin{abstract}
Sentinel lymph node biopsy (SLNB) is routinely used in the staging of invasive breast cancer. The aim of this study was to investigate the diagnostic accuracy of ultrasonography (US) compared to magnetic resonance imaging (MRI) in the pre-operative assessment of metastatic disease to the axilla in breast cancer patients at our community hospital. We retrospectively reviewed a prospectively collected database of 277 patients seen at our breast center from 2009 to 2010. Patients with invasive breast cancer were then evaluated for axillary metastasis. Lymph nodes were sampled using fine needle aspiration (FNAB) or core biopsy. Histopathology of the sentinel lymph nodes (SLN) or results of the axillary dissection were compared to US or MRI results. A total of 228 patients had invasive breast cancer. In these patients, 122 lymph nodes were sampled. Pathology proven metastases to axillary lymph nodes were found in 76 cases. Accuracy and sensitivity were higher in US than MRI in detecting metastatic disease to the axilla $(70.2 \%, 84.6 \%, \mathrm{P}<0.001$ and $60.0 \%$, $52.6 \%, \mathrm{P}<0.1$, respectively). US was more accurate than MRI at detecting metastatic breast cancer in the axilla in our community hospital. Axillary US should be a routine part of assessment of breast cancer patients.
\end{abstract}

\section{Introduction}

Once breast cancer is diagnosed, the presence or absence of metastatic disease to the axilla is the most powerful predictor of survival and has significant implications for the treatment choices available to the patient. ${ }^{1-3}$ The current standard of care to evaluate the status of axillary metastasis is sentinel lymph node biopsy (SLNB). ${ }^{2,4}$ In the presence of a positive sentinel lymph node (SLN), the standard of care is to perform a complete axillary dissection, usually during a second operation which is often associated with increased morbidity and cost, as intraoperative frozen section analysis is often inaccurate in determining the presence of metastasis in axillary lymph nodes. ${ }^{2,5}$

US and MRI are two modalities often used in the treatment of breast cancer patients, particularly in the pre-operative setting. US is a readily available and relatively inexpensive way to evaluate breast cancer patients and has been shown to have significant utility in the treatment of these patients. MRI is an expensive but effective tool that is also being used with increasing frequency in the evaluation of these patients. ${ }^{6,7}$

As these modalities are utilized in the preoperative setting, having an accurate tool for evaluation of precise axillary LN status will allow patients to be offered a one-stage operative procedure, or potentially allow some patients to forego completion axillary dissection. A recent randomized prospective trial suggests that some select patients may not need to undergo completion axillary dissection following a positive SLNB, making evaluation of the axilla with a reliable non-invasive method an increasingly important part of the care of patients with breast cancer. ${ }^{8}$

The purpose of our study was to assess the accuracy of axillary US compared to MRI at detecting lymph node metastases in patients undergoing pre-operative evaluation and staging for invasive breast cancer at our community-based hospital. This study was undertaken in an effort to reflect the care that most patients with breast cancer currently receive in the community setting, as most studies to date on these modalities come from specialized centers. ${ }^{9-11}$

\section{Materials and Methods}

We retrospectively reviewed a prospectively collected database of 277 patients who were seen at our breast center from 2009 to 2010 after institutional review board approval was obtained. All patients with breast cancer were evaluated before definitive surgery at our multi-disciplinary breast center. MRI and US of the axilla were obtained after consensus between the providers. Biopsy was then performed using US for suspicious nodes. Node status on US and MRI was determined by chart review of imaging reports and classified by key findings of morphology. The US and MRI findings were classified into 2 categories: positive (suspicious for malignancy) and negative (likely benign). Those male patients who had not had imaging of the axilla performed, and patients with axillary lymph nodes that had not been biopsied prior to initiating neo-adjuvant chemotherapy, were excluded from the study.
Correspondence: Gopal Chandru Kowdley, Department of Surgery, Saint Agnes Hospital, Baltimore, MD 21229, USA

Tel. +1.410.368.8491 - Fax: +1.410.951.4007.

E-mail: gkowdley@gmail.com

Key words: breast, axilla, cancer, imaging.

Contributions: SR, GK data acquisition, analysis, manuscript writing, editing, conception, design; MS, JB data acquisition and analysis.

Disclosures: no commercial interests for any of the authors listed.

Institutional Review Board approval has been obtained for this study.

Received for publication: 30 September 2011. Revision received: 22 October 2011.

Accepted for publication: 19 December 2011.

This work is licensed under a Creative Commons Attribution NonCommercial 3.0 License (CC BYNC 3.0).

(C) Copyright S.I. Ramirez et al., 2012

Licensee PAGEPress, Italy

Surgical Techniques Development 2012; 2:e4

doi:10.4081/std.2012.e4

For US, the criteria used to determine positivity of lymph nodes included: i) asymmetric or eccentric cortical thickening/lobulations; ii) loss or compression of the hyperechoic medullary region; iii) absence of fatty hilum; iv) abnormal lymph node shape; v) markedly hypoechoic cortex; vi) thickened cortices; and vii) increased peripheral blood flow. ${ }^{9,12,13}$ The criteria used for MRI abnormality were: a lymph node which was i) prominent, ii) large, iii) suspicious, or iv) whose cortex was thickened. ${ }^{14}$

Final pathological confirmation of lymph node status was obtained by US FNAB or core biopsy, SLNB, or axillary lymph node dissection. The MRI was directly compared to US at detecting metastatic disease to axillary lymph nodes obtained by the various nodal sampling methods. Diagnostic specificity, sensitivity, positive and negative predictive values, as well as accuracy of axillary US and MRI, were calculated. Pre-operative MRI guided biopsy of axillary lymph nodes was not performed on our patients. Statistical analysis was performed using Excel (Microsoft ${ }^{\mathrm{TM}}$ ). Pearson's $\chi^{2}$ test was used and $\mathrm{P}<0.05$ were considered statistically significant.

\section{Results}

A total of 277 patients were seen at our multidisciplinary center in the period 2009 to 2010 , of which 228 had pathology proven invasive 
breast cancer. In these patients, 122 lymph nodes were sampled. As shown in Figure 1, the most common primary breast cancer for which imaging of the axilla was performed was invasive ductal carcinoma (86\%) followed by invasive lobular carcinoma (14\%). Most patients had T2 tumors (44.3\%) (Table 1).

Pathology proven metastases to axillary lymph nodes were found in 58 (47.5\%) cases. There was agreement between US findings and the final pathological histology in 40 of 57 cases, and MRI and final pathology in 58 of 96 cases.

Table 2 and Figure 2 illustrate the diagnostic accuracy of US and MRI in our series. Overall accuracy was higher for US than MRI. In 33 cases, assessment of the axilla was carried out by both US and MRI in the same patients. Of these, the imaging results of both modalities agreed with the final pathological status in 22 cases by US and 20 cases by MRI (data not shown).

\section{Discussion}

It is well known that axillary lymph nodes are the most important prognostic factors in the evaluation of breast cancer patients..$^{1-3,15,16}$ Proper staging requires the examination and evaluation of the axillary lymph nodes. According to Aitken et al., axillary node clearance remains the gold standard for evaluating metastases. In the 1990s, SLNB emerged as a highly sensitive and accurate way of assessing the presence or absence of metastatic disease within the axilla, decreasing the need for complete node dissection as mainstay of therapy. ${ }^{2,4}$ As a minimally invasive alternative to axillary lymph node dissection, it became an acceptable way to stage breast cancers in clinically node-negative patients. A negative SLNB is 95$100 \%$ likely to indicate a negative axilla ${ }^{17}$ and has a false negative rate of $10 \%$ or less. ${ }^{18}$ US and MRI are imaging modalities that offer the possibility of accurately assessing the axilla in an even less invasive manner.

US is a simple and reliable method of distinguishing between malignant and benign lymphadenopathy. It allows for the real-time biopsy of suspicious nodes with US guided FNA or core biopsy at the time of examination. There are several criteria for differentiating malignant versus benign lymph nodes on US. Among the most important of these is the ratio between longitudinal and transverse diameter, morphology, asymmetry or focal cortical thickening, as well as increased vascular involvement as demonstrated by doppler flow studies. $^{2,3,14,17}$ Reactive lymph nodes are a common finding on US of the axilla, and typically appear round or oval in shape, ranging from 10 to $25 \mathrm{~mm}$, with a clearly visible hilum that is hyperecohic.
US is highly user-dependent. . $^{1-314}$ At our breast center, US biopsy is performed by general surgeons with a special interest in breast cancer along with an US technician. We find this combination of expertise is invaluable in delivering a high degree of quality and accuracy for our patients, and may minimize issues of interpersonal variation in the delivery of care.

MRI is also a non-invasive method of assessing lymph node status in breast cancer patients and is being used with increasing frequency. Criteria for abnormal lymph nodes has been outlined by Lernevall ${ }^{14}$ and include length to width ratio of less than 1.5 , eccentric enlargement with focal thickening of the cortex, and compression or absence of the hilus. They reported a sensitivity of $90 \%$ using size of lymph node greater than $5 \mathrm{~mm}$. MRI and its use in axillary nodal staging are constantly being improved, and techniques continue to be developed. ${ }^{12}$

In our study, we demonstrated that US was more accurate (70.2\%) than MRI (60.4\%) in assessing axillary nodal status using the criteria listed above. This accuracy rate is consistent with other large series using trained radiologists performed in centers where US is rou-

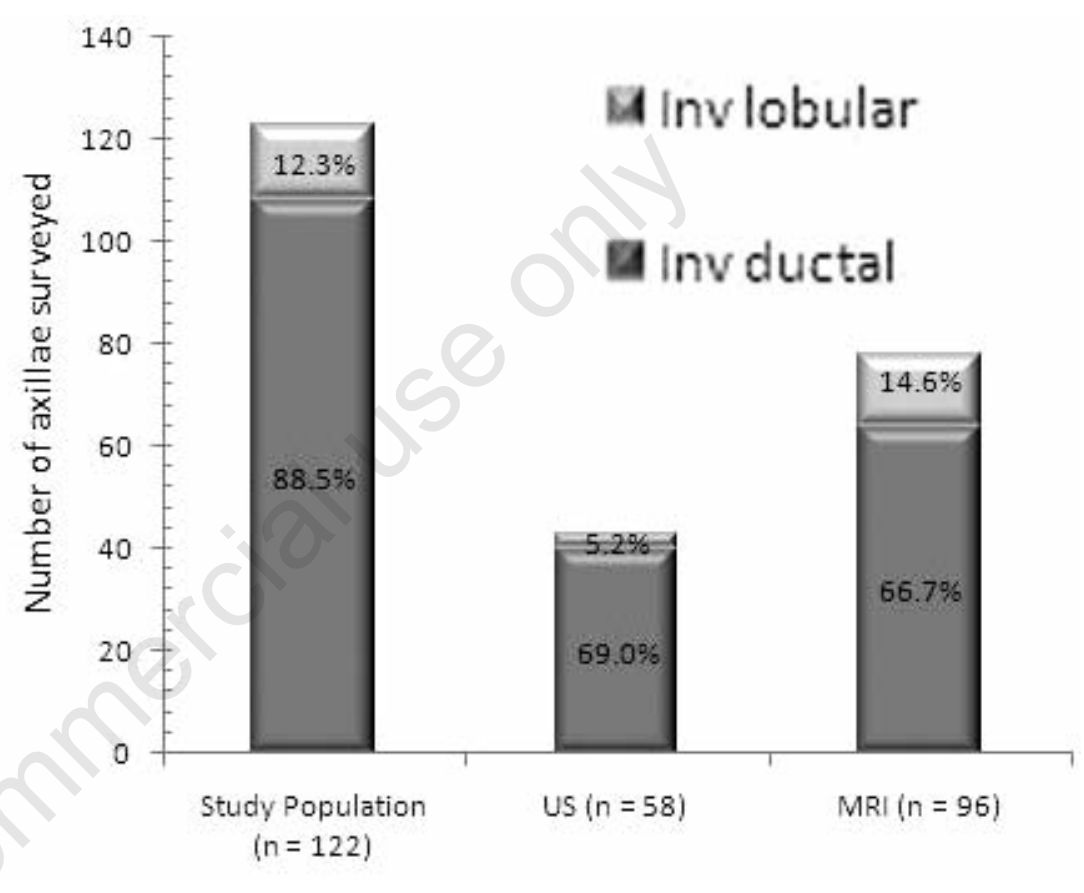

Figure 1. Primary tumor histology.

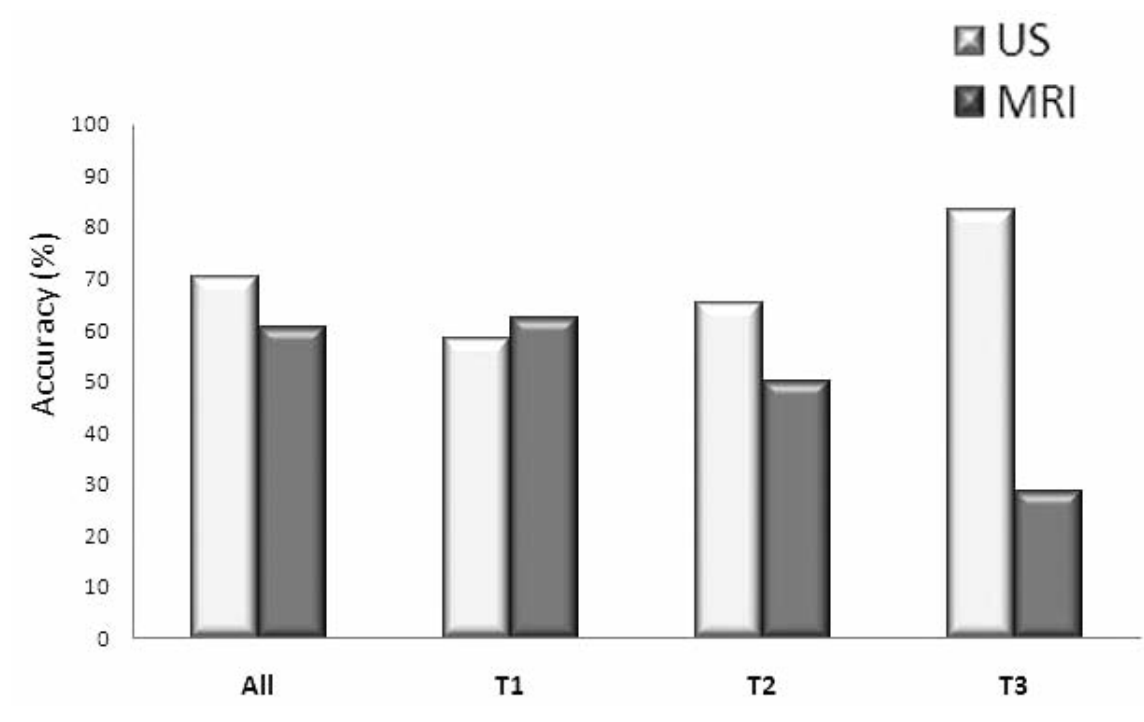

Figure 2. Accuracy of US compared to MRI at detecting metastatic disease to the axilla based on $T$ stage of primary tumor. 
tinely used in the pre-operative assessment of the axilla. ${ }^{9-11}$ Our study gave a sensitivity of $84.6 \%$ for US; slightly higher than that of previously published studies which report a range $56-72 \% .{ }^{19-25}$ We do use US in a selective manner at our hospital, as opposed to its routine use in all patients with breast cancer, and this may have some bearing on our more accurate results.

There is a direct relationship between prognosis and the number of lymph nodes involved with metastatic disease. In a study by Jakub et al. ${ }^{15} 449$ breast cancer patients with positive axillary SLN who underwent regional nodal dissection were examined to determine whether metastatic disease confined to the SLN had better prognosis than metastatic disease spread to non-SLNs. In this study, overall survival remained the same, independent of increasing number of SLNs involved, but decreased once disease extended to non-SLN. In contrast, recent data from Giuliano et al. ${ }^{8}$ would suggest that some patients with early

Table 1. T stage of tumor.

\begin{tabular}{lcc} 
T stage & N & $\%$ \\
T0 & 1 & 0.8 \\
Tis & 1 & 0.8 \\
\hline T1 & 43 & 35.2 \\
T2 & 54 & 44.3 \\
\hline T3 & 9 & 7.4 \\
T4 & 2 & 1.6 \\
\hline
\end{tabular}

Table 2. Diagnostic accuracy of axillary (A) US and (B) MRI.

\begin{tabular}{lcc} 
(A) & Path + & Path - \\
US + & 22 & 4 \\
US - & 13 & 18 \\
\hline Total & 35 & 22 \\
NPV & 81.8 & Alpha P $<0.001$ \\
\hline PPV & 62.9 & \\
Accuracy & 70.2 & \\
\hline SN & 84.6 & \\
SP & 58.1 & \\
\hline (B) & Path & Path - \\
\hline MR+ & 21 & 18 \\
MR - & 20 & 37 \\
\hline Total & 41 & 55 \\
NPV & 67.3 & Alpha P $<0.1$ \\
\hline PPV & 51.2 & \\
Accuracy & 60.4 & \\
\hline SN & 53.8 & \\
SP & 64.9 & \\
\hline
\end{tabular}

stage breast cancer may avoid axillary lymph node dissection altogether. This observation makes the assessment of axillary lymph nodes in the pre-operative setting even more important as accurately predicting the cancer status of the node may be as important as the removal and or identification of nodal status for the complete axilla.

A major limitation in our study was that only a few patients underwent both US and MRI. A total of 33 patients had both modalities performed. This may, in part, reflect the selective use of these imaging techniques at our breast center. Certainly, based on the data presented here, extension studies in which both these modalities are used in a prospective manner may better allow us to measure the true accuracy of US and MRI in our hospital.

In conclusion, the superior accuracy of US when compared to MRI in our study highlights the importance of including the ipsilateral axilla when performing US examination in a patient with breast cancer. US is readily available, faster, and less expensive than MRI, and serves as a non-invasive method of detecting axillary node metastases in women with invasive breast cancer. US may be performed with high accuracy at a community based breast center. Finally, given the increased morbidity associated with surgical axillary lymph node clearance, accurate pre-operative assessment of metastatic disease within the axilla may help reduce the number of operations and limit cost while optimizing the overall therapy.

\section{References}

1. Moore A, Hester M, Nam MW, et al. Distinct lymph nodal sonographic characteristics in breast cancer patients at high risk for axillary metastases correlate with the final axillary stage. Br J Radiol 2008; 1:630-6.

2. Nori J, Vanzi E, Bazzocchi M, et al. Role of axillary ultrasound examination in the selection of breast cancer patients for sentinel node biopsy. Am J Surg 2007; 93:16-20.

3. Luparia A, Campanino P, Cotti R, et al. Role of axillary ultrasound in the preoperative diagnosis of lymph node metastases in patients affected by breast carcinoma. Radiol Med 2010;115:225-37.

4. Noguchi M, Tsugawa K, Kawahara F, et al. Dye-Guided Sentinel Lymphadenectomy in Clinically Node-Negative and NodePositive Breast Cancer Patients. Breast Cancer 1998;5:381-7.

5. Sianesi M, Ceci G, Ghirarduzzi A, et al. Use of axillary ultrasonography in breast cancer: a useful tool to reduce sentinel node procedures. Ann Ital Chir 2009;80,
315-8.

6. Mortellaro VE, Marshall J, Singer L, et al. Magnetic resonance imaging for axillary staging in patients with breast cancer. $\mathrm{J}$ Magn Reson Imaging 2009;30:309-12.

7. Baltzer PA, Dietzel M, Burmeister HP, et al. Application of MR mammography beyond local staging: is there a potential to accurately assess axillary lymph nodes. AJR Am J Roentgenol 2011;196:W641-7.

8. Giuliano AE, Hunt KK, Ballman KV, et al. Axillary dissection vs no axillary dissection in women with invasive breast cancer and sentinel node metastasis: a randomized clinical trial. JAMA 2011;305: 569-75.

9. Sahoo S, Sanders MA, Roland L, et al. A strategic approach to the evaluation of axillary lymph nodes in breast cancer patients: analysis of 168 patients at a single institution. Am J Surg 2007;194:524-6.

10. Benson JR, della Rovere GQ; Axilla Management Consensus Group. Management of the axilla in women with breast cancer. Lancet Oncol 2007;8:33148.

11. Jain A, Haisfield-Wolfe ME, Lange J, et al. The role of ultrasound-guided fine-needle aspiration of axillary nodes in the staging of breast cancer. Ann Surg Oncol 2008;15: 462-71.

12. Mainiero MB. Regional lymph node staging in breast cancer: the increasing role of imaging and ultrasound-guided axillary lymph node fine needle aspiration. Radiol Clin North Am 2010;48:989-97.

13. Nori J, Bazzocchi M, Boeri C, et al. Role of axillary lymph node ultrasound and large core biopsy in the preoperative assessment of patients selected for sentinel node biopsy. Radiol Med 2005;109:330-44.

14. Lernevall, A. Imaging of axillary lymph nodes. Acta Oncologica 2000;39:277-81.

15. Jakub JW, Bryant K, Huebner M, et al. The number of axillary lymph nodes involved with metastatic breast cancer does not affect outcome as long as all disease is confined to the sentinel lymph nodes. Ann Surg Oncol 2011;18:86-93.

16. Verbanac KM, Min CJ, Mannie AE, et al. Long-term follow-up study of a prospective multicenter sentinel node trial: molecular detection of breast cancer sentinel node metastases. Ann Surg Oncol 2010;17:368-77.

17. Aitken E, Osman M. Factors affecting nodal status in invasive breast cancer: a retrospective analysis of 623 patients. Breast J 2010;16:271-8.

18. Cho N, Moon WK, Han W, et al. Preoperative sonographic classification of axillary lymph nodes in patients with breast cancer: node-to-node correlation with surgical histology and sentinel node biop- 
sy results. Am J Roentgenol 2009;193: 1731-7.

19. Bruneton JN, Caramella E, Hery M, et al. Axillary lymph node metastases in breast cancer: Preoperative detection with US. Radiology 1986;158:325-6.

20. de Freitas R Jr, CostaMV, Schneider SV, et al. Accuracy of ultrasound and clinical examination in the diagnosis of axillary lymph node metastases in breast cancer. Eur J Surg Oncol 1991;17:240-4.

21. Feu J, Tresserra F, Fabregas R, et al.
Metastatic breast carcinoma in axillary lymph nodes: In vitro US detection. Radiology 1997;205:831-5.

22. Sato K, Tamaki K, Tsuda H, et al. Utility of axillary ultrasound examination to select breast cancer patients suited for optimal sentinel node biopsy. Am J Surg 2004;187: 679-83.

23. Alvarez S, Anorbe E, Alcorta P, et al. Role of sonography in the diagnosis of axillary lymph node metastases in breast cancer: A systematic review. Am J Roentgenol
2006; 186:1342-8.

24. Hammer C, Fanning A, Crowe J. Overview of breast cancer staging and surgical treatment options. Cleve Clin J Med 2008; 75:S10-6.

25. Jakub JW, Bryant K, Huebner M, et al. The number of axillary lymph nodes involved with metastatic breast cancer does not affect outcome as long as all disease is confined to the sentinel lymph nodes. Ann Surg Oncol 2011;18:86-93. 